\title{
CONSERVATIVE TREATMENT OF POLIOMYELITIS
}

\author{
By MRS. W. BROWNE, B.Sc.(Physiotherapy) RAND. \& MRS. M. GOODMAN, B.Sc. \\ (Physiotherapy) RAND.
}

(A paper presented together with a practical demonstration at the Polio Postgraduate Course held in May, 1955, in Johannesburg).

$\mathbb{W}$ HILE treating polio patients at the Johannesburg General Hospital, we have tried to gain as much knowledge as possible of the disease and its treatment from various scurces. But it is from practical experience with the patients themselves, studying the individual peculiarities in their manner of walking, rate of progress, and so on, that one gains a real understanding of this fascinating and rewarding branch of physiotherapy.

We all learn the basic principles of the treatment of polio patients during the course of our studies, but these cannot often be applied without modification. In practice. hundreds of questions arise for which one can only find an answer by study of available books and articles, discussion with other people interested in the subject, and careful observation and judgment.

The following will be a description of the treatment which most patients are receiving at the Hospital, and a discussion of some of the difficulties which may crop up. We do however realise that our knowledge of the subject is incomplete, and that some of the points mentioned may be controversial.

As soon as a patient is put under our care, we start a case history file for him in addition to the hospital cards which are too small to hold enough detailed information for these long term patients. The early history of the patient is taken down, together with the symptoms at onset, previous treatment and so on. After careful observation of the patient during two or three treatments, it is possible to add accurate notes on the present condition of the joints and muscles. This procedure gives the physiotherapist a clear picture of the patient's condition; and further has the advantage of providing a written record of the patient's progress, to which both patient and physiotherapist can refer for encouragement when a static period in the treatment seems to have been reached.

It is essential that the physiotherapist should determine the cause of the various abnormal conditions. The inability to dorsiflex the foot, for instance, may be due to total paralysis. The same symptom however, may be found when spasm of the antagonists is present, or in the case of mental alienation. Each of these causes will require different corrective treatment. In the same way it is important to note any existing deformities or faults in walking, and ascertain whether they are due to spasm, paralysis, or in long standing cases, just due to habit.

One must not be satisfied with an examination only at the commencement of treatment, but should be continually on the look out for changes in the patient's condition. One thus notes the progress of the patient and more important, can discover if any adverse changes have developed.

It is important to gain the co-operation of a patient. New patients should be made to understand that although the prognosis is always uncertain, a lot depends on their will to improve. co-operation with the staff, and their determination to practise, even when not under supervision; that a muscle will only increase in size if worked to a maximum, and that we can often strive for, and obtain a well-functioning limb, even if a certain muscle never regains full stength.

But in spite of all this encouragement to the patient, the physiotherapist herself must take a lot of responsibility during the treatment. She should avoid interruptions and general discussions with the patient and both she and the patient should concentrate hard on the attempted movement, more especially if there is very little movement present. The physiotherapist is also responsible for varying the routine, giving bed and standing exercises, weights and free movements. She should also encourage progression wherever possible.

Ball and pole exercises, pulleys, springs, and occasional class treatments, add interest to the work, but should not be given as a substitute for basic corrective exercises. Occupational therapy combined with physiotherapy has proved valuable as well as interesting to most of the patients. When patients are warded, we plan as full a programme of activity as possible. We treat them when possible in the out-patients' department and encourage them to get dressed rather than to shuffle around in a gown and slippers.

We now come to the actual treatment of these patients. In the fever hospital they receive hot packs, passive movements, and later active movements.

Sub-acute stage:

After examination, the full programme of treatment for the patient is worked out. As the exercises can best be demonstrated on the lower limb, we shall deal mainly with this part of the body in our talk.

\section{Passive movements:}

Every effort is made to overcome the spasm by stretching the affected part. This stretching can be taken to the point of discomfort, but should not be allowed to cause acute pain, as this will only increase the spasm, and actual tearing of the muscle fibres may occur. It is advisable to apply heat where spasm is severe. Tightness of plantarfiexors is commonly seen in polio patients. The advisability of stretching these muscles by the usual method has been questioned, as the stretching effect would be concentrated on the intrinsic muscles of the fooi. A better method, and one which needs less effort, is to grasp the heel with the hand and push the foot up with the fore-arm. The other hand fixes the leg above the ankle joint. The patients can also help themselves to stretch these muscles. A good method is for them to stand bare-footed on the floor when they get up in the morning, while their muscles are still warm. When they can get the affected heel on the ground they should bend their knees, thus causing a greater stretch on the calf muscles. In some patients certain muscle groups respond better to a slow stretch which is gradually increased to a maximum; whereas other muscles may loosen up better with a rhythmical stretch.

Heat:

We seldom apply heat to the patients who come for treatment, but in severe cases of spasm, it can be of value for relieving tension. During cold weather, patients whose extremities are affected will work much better when these parts have been warmed. Dry heat can be applied, but it is better to immerse the limb in water, as the patient can work the muscles to best advantage in this medium. It appears that some patients will retain spasm in several of their muscle groups for months after onset of the disease, in spite of intensive treatment from the beginning. In such cases, heat as well as stretching manipulations should be used. Patients are encouraged to take hot baths at home, and to do the stretching exercises in the bath when the muscles are warm and relaxed. 


\section{Exercises:}

We now come to the fundamental and most important part of the treatmen - the actual exercises to strengthen the muscles. We must always bear in mind that the harder we work a muscle the better are its chances of improving, and it is important to stress this fact to the patient. We must realise, however, that we have to treat muscles of varying strengths, and deal with the situation accordingly. When confronted with a muscle in which not even a flicker of movement is observed, we must move the joint passively, make the patient concentrate on the movement, and tell him to try and do it himself. It is just possible that he cannot contract the muscle because of mental alienation, that is, because the organisation of impulses from the brain to the muscle has been interrupted. In this case there is only apparent paralysis, and it is our duty to try and re-establish the pathway of impulses between the brain and the muscle. If the movement is lost because of nerve cell injury, we should try to prevent this mental alienation from taking place, in case recovery of some of the nerve cells occurs. We can attempt this in any of three ways, according to the patient's response. We can perform a movement and ask the patient to try and work with us; we can ask the patient to try and resist the movement we are performing; or lastly, the patient can try to hold the limb in a position where gravity exercises an opposite pull.

Once a flicker is present one can go ahead with active re-education. Now the patient can try to move actively and hold the muscle in the contracted position. Assistance can be given to complete the movement, but the patient does as much as possible on his own. As the muscle strength improves, resistance is given, and more exercises are included until the muscle is normal. As soon as the patient is able to do it, he can lift weights, ride the bicycle, and use other apjaratus on his own. In the early stages and when treating very weak muscles, the physiotherapist must be with the patient during the whole treatment, but, as the muscle groups increase in strength, the patient should be encouraged to work on his own before or after the treatment time. He can, for instance, do balance exercises, or lift weights with his quadriceps. On the whole manual resistance is preferable to that of springs and weights, especially in the early stages, when the muscle may work better in one range than another. Manual resistance can be varied, whereas if a spring were given, it would have to be weak enough to be pulled in the weakest range of the muscle.

For most of the basic exercises the lying or prone lying positions are used, as the body is well supported and steady, and few muscles have to be used to maintain the position. In these positions, the origins of many of the muscles are fixed, allowing the inserting ends of the muscles to work without hindrance. It is, however, impossible to be dogmatic about this, as it is remarkable how the optimum position for the performance of a certain movement varies from patient to patient. For example, one person may find that he can dorsi- and plantar- flex his foot best in the lying position, while another may get the feeling better in the prone lying position with the knee bent at a right angle; a third person may find it very awkward to move the foot in either of these positions and prefer to do it by crossing one leg over the other in the sitting position and working with gravity eliminated As the treatment progresses all the positions should become equally easy but in the early stages we have found it advisable to use the optimum position, as this provides the maximum amount of exercise for the muscle.

We try to exercise each muscle group in three or four different ways during the treatment. This can be done by giving eccentric, concentric or static movements, by altering the starting position, or varying the type of resistance given. This variation adds interest to the treatment, and one can also be more sure that all the parts of the muscle do work, because if some fibres do not respond to a certain
type of movement, they may respond to another. This also enables one to work a muscle to fatigue in one position. give it a rest by exercising other groups and then to retur. to the muscle with a different exercise. The following some of the exercises which could be given to weak ham string muscles.

1. Prone lying. Attempt to balance leg which is flexed to $90^{\circ}$ at the knee.

2. As a progression, the physiotherapist can push the leg just off balance while the patient tries to recover the

3. Prone lying. Knee flexion concentrically. Assisted free or resisted movement can be given as required.

4. Prone lying. Knee flexion eccentrically with gravity resisting or manual resistance as required.

5. Lying. Thigh supported in sling with hip flexed. Spring attached to foot from a point above the knee. Knee flexion against the spring concentrically or eccentrically.

6. Lying. Drawing the knees onto the chest against the therapist's resistance, concentrically or eccentrically.

We would here like to emphasise that at the end of a patient's treatment, the muscles should be so fatigued that it would be almost impossible for him to do any more without a rest. This fatigue is not at all harmful as subsequent increase in muscle strength will show.

\section{Guthrie-Smith Apparatus:}

There has recently been a fair amount of discussion at the Hospital on the value of the sling suspension ap paratus in the treatment of polio patients. When abused, this method of treatment can be a total watste of time and effort, but when properly used it can be of great value. for no other method provides so simple a means of giving assistance to a movement, while at the same time, with resistance given by the physiotherapist, hard work is provided for a weakened muscle. We never suspend a patient's limb and tell him to get on with an exercise, leaving him to swing it aimlessly, but instead supervise the movements, giving resistance wherever possible.

As an example of the uses of the apparatus we will take the abductor group of the leg and see how in various stages, slings can be used in its treatment.

1. Should a patient have stiff abductor or adductor muscles a rhythmical swing of an axially suspended limb will help to ease this.

2. In a severely paralysed patient who has not regained the "feel" of the movement, the limb can be suspended and moved gently in and out, while the patient is told to think about the movement and to try to perform it.

3. As the patient progresses, he can swing the limb freely in the sling. The physiotherapist can at intervals and without warning the patient, suddenly instruct him to stop the movement and to hold the limb in the abducted position.

4. Finally, the physiotherapist can take up a position to resist the limb, and this resistance can be given concentrically or eccentrically. When paralysed muscles are put on the stretch, this acts as a stimulus, and a better contraction will result. This fact should be made use of in sling-suspension exercises and muscles should on the whole be worked from their fully stretched position.

\section{Hubbard Tank:}

We have made considerable use of the Hubbard Tank for carefully selected cases during this polio epidemic. It is useful when severe paralysis is present or where there is much muscle spasm, and passive movements are given in this medium. The warmth and buoyancy of the water relieve the tension of the muscles and increase the mobility 
of the limb. In very severely paralysed patients it is worth whe tank even if only for the psychological wh on bed-ridden patients. A selection of effect which it has in the tank, and the patient is made to exercises is given in the the should only prescribe this work as hard as possible. Ona consideration, as it is treatment to patients operation, and the time may be better rather a tedious operation, and

swimming:

The disadvantage of using apparatus e.g. bicycle whill use the unaffected muscles to do the work and not the weak muscles. To a certain extent the same may be said weak mimg although the advantages of this exercise are of swimming, although few seasons, we have been taking the many. For the past few to the University swimming bath Hospital polio patient they all get together and a very happy twice a week. Her th far as the swimming is concerned. atmosphere prevails. As far as the swimming is concer own. we try to get them to swim as far as possible on their own. and in addition, to move the affected part legs are affected. in the water. For example, a patient whow end of the bath, will start by walking across the shallow end of the bath, and the number of times he walks will be progressed from time to time. For a very weak patient, this is a good exercise as although the water buoys the body, and the supporting muscles of the legs do practically no work, it offers resistance to the forward movement of the legs and can be quite fatiguing. After a short rest the patient will exercise his legs in the swimming position. He can perform a kicking motion with the legs when in a prone position and holding on to a board. This is excellent for the gluteal muscles, while the same movement performed on the back will exercise the hip flexors. The breast stroke is an excellent exercise for the adductor of the hip while a new form of the butterfly stroke, where the legs are fiexed and then vigorously extended, works the quadriceps very strongly. Where the hip extensors are very weak, and the limb is heavy, it may be necessary to tie an inflated bicycle tube around the hips, to prevent the legs from sinking.

To exercise an arm which can be moved out of the water, the crawl and back stroke movements can be used. For a patient who had a weak anterior deltoid and pectoralis major, we used to stand in the water, and support him lightly under the back. He would then lift the arm up and out of the water over and over again. This markedly strengthened the muscles, but could, of course, have been done equally well on a plinth. In fact, in the latter position the shoulder girdle would be fixed and a truer movement would be cbtained. Yet the extra interest which the swimming gave the patient made him persevere.

All the patients need very careful observation during the swimming exercises, or else they will just rely on the lift of the water and their unaffected muscles to produce movement.

One of our patients who had a generally affected left arm, and who was quite unable to lift it out of the water, was made to do breast stroke. To keep his limb elevated and away from his body, we tied a slightly inflated bicycle tube over his shoulder, and twisted it once around his wrist. This slight support enabled him to exercise his arm most effectively, and though he went only at a snails pace, he used to swim three or four hundred yards at a time. It is in cases like this, where all muscles in the limb are affected to more or less the same degree, that swimming is of value, whereas in a limb with some strong muscles and some weak ones, swimming will probably benefit only the strong ones. In spite of this, swimming can be of definite value and is an excellent means of improving the general body musculature of these patients.

Walking:

From the earliest stages of treatment we start reeducating the patient's walking where leg or trunk muscles are affected. This is a most important aspect of the treatment and, if neglected, can result in a most ungainly and fatiguing walk. At first the patient may just be ignorant of the way he is walking, or uncertain of the ways he can improve this; but it soon becomes habitual, and even when muscle strength improves, the gait may remain awkward. Naturally, we cannot expect a patient to walk correctly when certain of his muscles are paralysed, but we can ensure that he walks to the best of his ability.

First of all, it is important to see that the patient's feet are properly supported. He should wear well fitting shoes and socks. High heeled shoes, very open sandals and thick crepe soles should not be permitted. The stiff leather shoes which one sometimes sees, allow very little movement and exercise of the toe and intrinsic foot muscles, while a shoe which is too heavy will impede the walking of very weak patients. Calipers should only be used when absolutely essential to the patient, such as in the case of complete loss of the dorsiflexors of the foot, and they should be discarded as soon as possible. If they are used when the muscles themselves could work, they relieve the muscles of their natural function and these will then deteriorate. The same applies to the use of the crutches and sticks. In the early stages it is preferable to allow the use of crutches rather than to allow the patient to walk in an ungainly way. There is a tremendous difference between the support offered by crutches and that given by sticks, as the latter are really comparatively unsteady; but as soon as possible progression should be made to sticks and later to having no support at all.

We never start re-education of walking in the wheelcrutch, as this does not offer the necessary support to a weak patient. Instead of being able to concentrate on his walking, he is always conscious of the fact that he must prevent the wheel-crutch from running waay from him. The parallel bars provide an ideal piece of apparatus for the re-education of walking. They offer a steady support for a sufficient distance to enable several steps to be taken. In addition, they provide a suitable means of support fos standing exercises. As soon as possible the patient is taught to walk with crutches. At each treatment, his walking should be watched by the physiotherapist so that his faults can be corrected. He should also walk in front of a mirror to learn to recognise and to correct his own faults, e.g. limping, uneven timing, or uneven spacing of steps.

The following are a few exercises which are given to the patient in the parallel bars.

1. Stride, standing. Balancing without holding on.

2. Stride, standing. Transference of weight from one leg to the other.

3. Stride, standing. Two arm bend and stretch in all directions.

4. Stride standing. Catching and throwing a ball.

5. Kicking a ball without holding on.

6. Walk standing. Transference of weight from one leg to the other.

7. Standing. Free leg swinging forward and backward. This exercise encourages the patient to use his ankle dorsiflexors to keep his foot from hitting the floor.

8. Standing. Bending the knee slightly, then attempting to straighten up against gravity.

9. Standing. Attempted raising on to the toes. Thi: is progressed to Heel Raise, Knee Bend.

10. Standing. Marking time, progressed to stepping over sand bags.

11. Half standing, with knee unlocked, to exercise the quadriceps. 
Most of the polio patients have a fear of falling when they start to walk, and in fact often do fall. The following exercise gives them more confidence, as they learn to regain their balance when this is upset, thereby lessening their chances of falling. In addition, they learn not to be frightened at the thought of a fall, as this fear often makes them over cautious and prevents them from walking to the best of their ability. They should also learn to get up after a fall.

Exercise: The patient stands in the stride position on a large mattress. The physiotherapist then tries to push him off balance, first sideways and then forwards and backwards, while the patient resists this. When the patient falls he is taught to get up again.

As soon as possible, patients are taught to walk up and down steps, and up and down sloping surfaces. We are lucky at the General Hospital to have a very convenient ramp just outside the Gym, so that patients soon learn to overcome this rather difficult obstacle.

I would now like to deal with four topics-mental alienation, inco-ordination, trick movements, and the relative value of certain important muscle groups to the patient.

\section{Mental Alienation:}

One of the biggest problems we have to cope with in the treatment of poliomyelitis is that of mental alienation: a patient who has temporarily lost some or all of the power in a muscle, tends too lose the ability of voluntary activation of that muscle, even though the nerve cells controlling the muscle may have recovered, that is, he has forgotten how to contract the muscle. The treatment of this condition has already been dealt with.

\section{Inco-ordination:}

One also commonly finds inco-ordination in polio patients. Co-ordination is the working together of various groups of muscles to perform a desired movement with the minimum expenditure of time and energy. If one muscle of a group which usually act together is lost, inco-ordination will result, e.g. if the quadriceps are paralysed, the natural movement of walking is upset, and the patient has to re-organise his movements to accommodate for this loss. He may do this by swinging his leg forward without bending the knee, and locking the knee mechanically without using the extensors, when he takes his weight on this leg. Unless this situation is watched for and corrected, the patient may habitually walk in this inco-ordinated way, even when muscle power has returned, i.e. he has learnt to do without a certain muscle, and must be taught to use it again.

\section{Trick Movements :}

A third very important point which I wish to discuss is the question of trick movements. A trick movement occurs when a patient produces a similar movement to the one desired without using the affected muscle at all, or without using it to its best ability. It usually occurs when a patient is asked to perform a movement which is difficult or impossible for him to do. He subconsciously substitutes another movement in his desire to perform the action. The physiotherapist must always be on the look out for these movements. Some are obvious, while other more obscure ones are most difficult to spot. They all result in the patient not working the required muscle, and must be rigidly prevented. The simple mechanism of these trick movements should be explained to the patient, and he himself should learn to spot when he is making a mistake. In fact, long standing, well trained patients can usually spot these movements as soon as they occur. A few of the common trick movements are:

1. Half-lying. Ankle-dorsi and plantar-flexion. The patient uses his trunk side flexors to produce a hip updrawing movement. With the heel as a fixed point on the bed, the trick movement is produced.
2. In- and eversion of foot. This is produced by interna and external rotation of the hip.

3. Lying, leg axially suspended in a sling. Hip abduction Trick movement is produced by the trunk side flexors.

4. Lying, leg axially suspended. Hip abduction. Patient externally rotates the hip and then performs the abduction movement. This movement is thus performed or aided by the hip flexors.

5. Prone lying. Knee flexion, done by flexing the hip Mechanism: The hamstring muscles pass over two joints, and can extend the hip and flex the knee. If the hip flexors should contract and flex the hip, the hamstrings are put on the stretch, and by mechanica action, the knee will be slightly flexed without muscular contraction.

6. The same action can be seen in extension of the knee If the hip is extended a stretch is put on the quadriceps, and extension is facilitated.

7. Prone lying. Knee flexion. When the hamstrings are weak, the action of gravity can be decreased by internally rotating the hip, and letting the leg fall slightly sideways.

Value of Muscle Groups:

Before closing, I would like to mention the relative value of three muscle groups of the leg in different everyday activities. Standard anatomical text books mention the main actions of muscles without elaborating on the various conditions under which they function. This I feel leaves an unfortunate gap in our knowledge.

The hamstrings, for example, while smaller and less powerful extensors of the hip than gluteus maximus, are much more useful for ordinary purposes of life, as they act normally in walking and standing, while the gluteus maximus does not. Any normal person can prove this to himself by feeling the gluteus during walking, as it remains relaxed. The result is that one who has lost the use of his gluteus maximus may stand and walk normally, while one who has lost the hamstrings can stand and walk only by throwing the weight of the trunk so far back that it tends to overextend the hip. This is done to prevent his falling forwards as the hamstrings only, and not the glutei, are able to counteract gravity in this position. A patient whose gluteus maximus is absent is still able to do straight leg raising in the prone lying position. The hamstrings can be felt to contract on any normal person doing the above exercise.

The hamstring muscles are also of utmost importance in the stability of the knee. It is thought by some that when the knee is locked in the standing position, it forms a rigid support without muscular activity, and that hyperextension is prevented by the various ligaments. But 1 clinical observation demonstrates that the hamstrings act as important ligaments of the knee and without them the leg will hyper-extend progressively. This hyper-extension will eventually overcome the resistance of the cruciate, co-lateral, and posterior ligaments, which then stretch rapidly and progressively.

The quadriceps are often affected in polio, and this can result in a severe disability to the patient. The patient can stand erect without difficulty because of the tendency of the weight of the body to hyper-extend the knee. Such persons can walk, provided that they avoid flexing the hip far enough to cause flexion of the knee by the weight of the leg and foot. They do this by taking very short steps which they can lengthen somewhat without danger by swinging the hip forward as far as possible with each step, giving them a waddling gait. Alternatively, they can swing the leg forward with force, locking the knee when the heel touches the ground. If the patient tries to hurry or swing the foot too far forward he will fall. Running, jumping, and so on are of course quite impossible.

Although the hamstrings have been mentioned as being of prime importance in walking, standing, etc., when any force is required in extension of the hip, the gluteus 
naximus is essential. Movements, such as standing up from the sitting position, jumping, running, etc. are impossible without it.

Whereas the hamstrings act when the hip joint is almost straight, the gluteus maximus comes into play the angle of the hip is more than $45^{\circ}$. We have all noticed how cyclists stoop forward, how old people stoop to climb up steps, and that sprinters start a race in the crouching position. These manoeuvres all aim at getting the hip at the required angle for gluteus maximus, the strongest hip extensor to come into play.

In spite of having strong back extensor muscles, a atient with a paralysed gluteus maximus cannot arch his back in the prone lying position as the pelvis cannot be fixed.

Lastly, I would like to mention the importance of all the muscles passing over the ankle joint, for balancing the body; when standing, we are continually changing the position of our bodies in relation to the centre of gravity, and it is mainly the muscles of the foot which are responsible for bringing the body into line again.

In concluding, I would like to mention that polio patients should, if possible, be treated by one physiotherapist for a considerable period of time. Several weeks are needed before the patient and therapist can work together really effectively towards a common goal. After many months, however, a change of physiotherapist may do the patient a world of good, as a fresh approach and new ideas and exercises almost invariably act as a tonic.

Finally, we must realise that the treatment of polio patients still leaves much scope for improvement. We should continually be on the alert to learn new ways and methods of treatment which must inevitably develop with the progress of medical science.

\section{When Ionization is indicated...}

\section{Imadyl Unction 'Roche'}

is the substance of choice.

\section{Containing $1 \%$ histamine-dihydrochloride}

it will produce local effects only and

will not cause general vasodilation.

Imadyl 'Roche' is freely available
again from

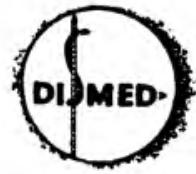

Medical Distributors

Specialists for Physical Medicine Appliances

P.O. Box 3378

JOHANNESBURG

Telephone 23-8106

236, JEPPE STREET

\section{OBITUARY}

Rhoda Valerie Marks, daughter of Mr. and Mrs. $\mathbf{H}$. Marks of Kensington, Johannesburg, was tragically killed on 27th July, 1955.

She was born in Johannesburg and was educated at Jeppe High School, and from there she went on to study Physiotherapy at the Witwatersrand University from which she graduated in 1949.

Rhoda worked for a while at the Johannesburg General Hospital and Coronation Hospital before going to Crown Mines where she was employed at the time of her death.

Having spent four months' holiday in Europe, she was returning home when the plane in which she was travelling crashed in Bulgaria. She was buried in Israel together with the other victims of the disaster.

Not only was Rhoda an enthusiastic and able student and a credit to her University, but she was an extremely loyal and active member of the South African Society of Physiotherapists, and we shall always remember her as a good friend and an outstanding physiotherapist. Her passing is deeply mourned, not only by her individual friends, but by the Physiotherapy Society as a whole.

\section{CONFERENCE}

Since several matters of importance have arisen recently which must be discussed by representatives of all Branches of the Society it has been decided that a Conference must be held this year.

To minimise costs, this will be held in Johannesburg on October 8th, 9th and 10th. Details are given below, and members are reminded that they will all be welcome to take part in the business discussions as well as the social functions, though only delegates elected by the Branches are entitled to vote.

\section{DRAFT PROGRAMME}

SATURDAY, 8th. 9.00 a.m.

Opening Speech and Welcome by Dr. K. Mills, Medical Superintendent, Johannesburg General Hospital.

10.00 a.m. Visits to various hospitals.

2.00 p.m. Business.

4.00 p.m. Tea.

4.30-5.30 p.m. Business.

7.00 p.m.

Cocktail Party.

SUNDAY, 9th.

a.m.

1.00 p.m.

2.00 p.m.

4.00 p.m.

$4.30-5.30$ p.m.

Evening

Native War Dance.

Lunch.

Business.

Tea.

Business.

Film or Music.

MONDAY, 11th.

9.00 a.m.

11.00 a.m.

11.30 a.m.

1.00 p.m.

2.00 p.m.
Business.

Tea.

Business.

Lunch.

Business.

Tea. 